\title{
PAEDIATRICS
}

\section{Understanding pubertal precocity-are kids growing up faster?}

Pubertal data are an integral part of monitoring health trends in children. Two recent studies have shown that children are entering puberty at younger ages than have been historically noted. Furthermore, much variability is evident between individuals as to what the defining features are of the pubertal transition.

The first study, led by Annette Mouritsen, had a longitudinal design (over 5 years) and repetitively analysed serum samples from 179 healthy Danish children (89 girls and 90 boys). The researchers found that only a minority of girls (7\%) and boys (25\%) experienced pubarche (attainment of pubic hair) as the first sign of puberty. Instead, girls predominantly developed breast tissue as the first sign (78\%), with boys experiencing testicular growth (59\%). In this cohort, girls were approximately 10 years old when these signs became evident, and boys were 11 years old.

"We also observed great interindividual and intraindividual variability in circulating levels of adrenal hormones, such as adione, during the pubertal transition," explains Mouritsen. Despite the variability, increased circulating adione was found to be associated with pubertal onset; the increase was more pronounced in girls than in boys. A similar increase was not observed in the androgen dehydroepiandrosterone. Finally, body composition (including BMI) was also not associated with the onset of puberty in either girls or boys.

The second study, conducted in the USA, found that puberty in boys occurs earlier (by the tenth birthday for the onset of testicular growth and by age 10.5-11.5 years for the onset of pubic

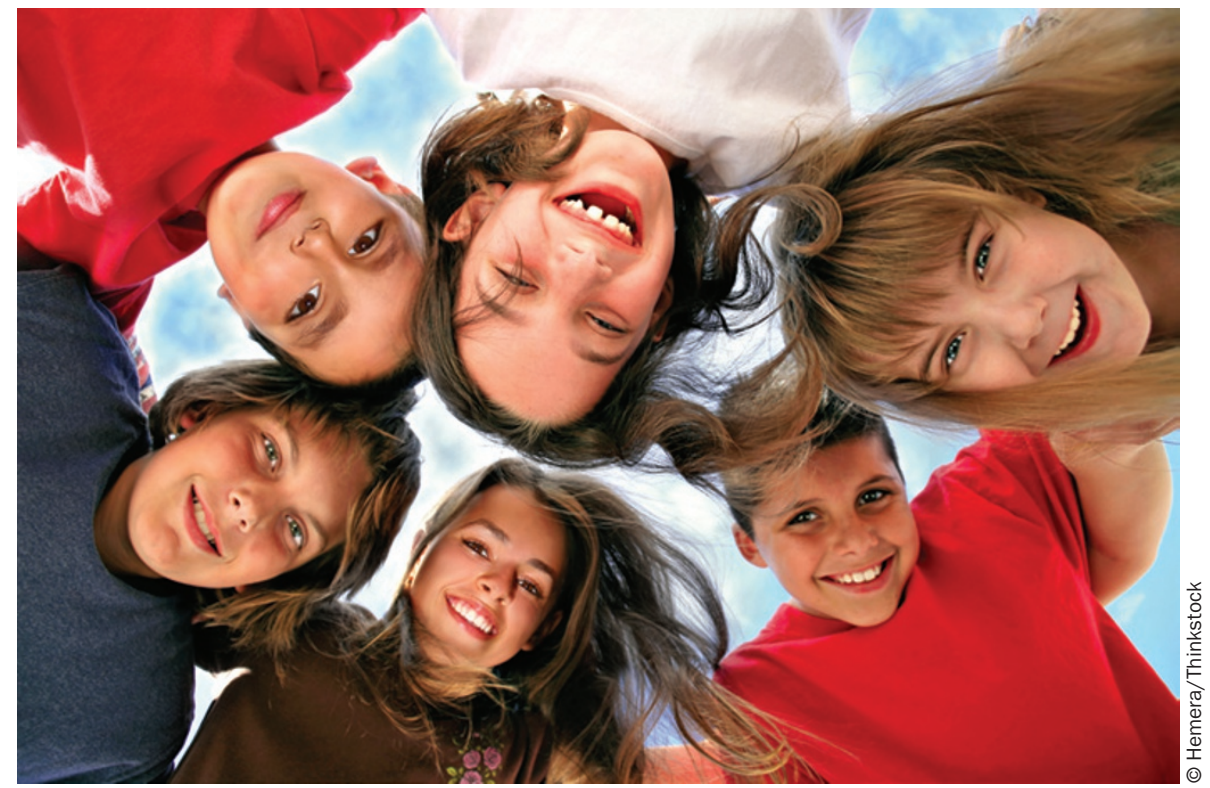

hair growth). Interestingly, black children showed earlier pubertal onset than their white or hispanic counterparts. "We don't know why these differences exist," says lead investigator Marcia Herman-Giddens. "Although racial differences in growth and developmental markers are known, these differences might be influenced by environmental factors."

These studies both highlight an important unmet need in clinical practice. That is, if we are unclear as to when puberty should occur and what features best define its onset, how can children be evaluated and treated for pubertal precocity or delay? Furthermore, if children are developing sexually at an increasingly early age, social and cognitive issues will emerge that will require concerted efforts to address.
"The gap between the onset of physical development in boys and the maturity of the brain is concerning," adds HermanGiddens. Consequently, a re-evaluation of sexual education programmes might be necessary. Ultimately, further research is needed to unravel the nutritional, environmental, genetic and epigenetic contributions to pubertal onset before such steps can be taken.

Mina Razzak

Original articles Mouritsen, A. et al. The pubertal transition in 179 healthy Danish children: associations between pubarche, adrenarche, gonadarche and body composition. Eur. J. Endo. doi:10.1530/EJE-12-0191 | HermanGiddens, M. E. et al. Secondary sexual characteristics in boys: data from the Pediatric Research Office Settings Network. Pediatrics doi:10.1542/peds/2011-3291 